\title{
Um framework para ecologia da aprendizagem na cultura digital
}

\author{
Pedro David Netto Silveira ${ }^{1}$, Davidson Cury ${ }^{1}$, Crediné Menezes ${ }^{1}$ \\ ${ }^{1}$ Departamento de Informática \\ Universidade Federal do Espírito Santo (UFES) - Vitória, ES - Brasil \\ \{pedro.dns, dedecury, credine\}@gmail.com
}

\begin{abstract}
Education has changed a lot in recent times, inside and outside the traditional school, where only part of the construction of knowledge happens. Learning occurs in everything we do and comes from the interactions carried out within our cognitive ecologies, which can be fostered to improve the learning constructivist process. In this article we present an overview of a small cut of current state of education and where we believe it goes, following the idea of learning without frontiers. Within this context, we also suggest a conceptual architecture of a ubiquitous environment to enhance learning.
\end{abstract}

Resumo. A educação mudou muito nos últimos tempos dentro e fora da escola tradicional onde acontece apenas uma parte da construção do conhecimento. A aprendizagem ocorre em tudo o que fazemos e advém das interações realizadas no âmbito de nossas ecologias cognitivas, que podem ser fomentadas para melhorar o processo construtivista de aprendizagem. Neste artigo apresentamos um pequeno recorte do panorama do estado atual da educação e para onde acreditamos que ele caminha, seguindo o pensamento de uma aprendizagem sem fronteiras. Dentro desse contexto, sugerimos ainda um framework conceitual relativo a um ambiente ubíquo para potencializar a aprendizagem.

\section{Introdução}

O mundo tem vivido uma mudança de paradigma educacional. As salas de aulas, com horários fixos, estabelecidas em uma determinada região geográfica, estão perdendo espaço para ambientes virtuais onde ocorre aprendizagem cooperativa, em nossa sociedade onde a cultura digital é cada vez mais presente.

As tecnologias inteligentes já transformaram muitas partes de nossas vidas, como na movimentação do nosso dinheiro em instituições financeiras ou simplesmente como agendamos e chamamos um táxi. Não há dúvida de que a Inteligência Artificial (IA) também influencia significativamente o que ensinamos e aprendemos, bem como a forma como o fazemos.

Vivemos uma grande oferta tecnológica no que diz respeito à IA, com surgimento de aporte de novas tecnologias digitais, com aumento significativo de demandas de usuários por acessibilidade, interoperabilidade, usabilidade etc., e mesmo assim ainda existe a dificuldade de se aprender como o apoio das tecnologias convencionais. Portanto, novas concepções tecnológicas, que suportem novas pedagogias, são inestimáveis. 
VII Congresso Brasileiro de Informática na Educação (CBIE 2018)

Anais do XXIX Simpósio Brasileiro de Informática na Educação (SBIE 2018)

Nos últimos anos, a partir da ciência das ecologias cognitivas, temos dirigido nossos esforços no entendimento de que aprendemos em tudo o que fazemos, em todas as atividades na quais nos envolvemos, e que elas podem ser conectadas para aprendermos mais. Para facilitar esse processo, contamos com o aporte ubíquo da tecnologia, não apenas para nos inserir dentro dos diversos contextos, mas para ser um alicerce onipresente que nos auxilie a: (i) evitar tarefas desnecessárias, (ii) ajudar na captura daquilo que nossos sentidos podem requerer, (iii) perceber em quê devemos nos focar dentro de um contexto onde muitas coisas acontecem ao mesmo tempo e (iv) registrar, buscar mais informação, nos lembrar posteriormente etc., daquilo que está sendo aprendido.

Fazer tudo isso exige ferramentas inteligentes que devem ficar a serviço de quem aprende. Essas ferramentas devem compor um ambiente cujo principal objetivo é potencializar a aprendizagem cooperativa facilitando as interações, partindo do princípio que a inteligência ou cognição são o resultado de redes complexas onde interagem um grande número de atores humanos, biológicos e técnicos. [Lévy 1999]

Dentro desse contexto, nosso objetivo é apresentar um estudo para evidenciar uma aprendizagem sem fronteiras, que reúne ideias de outros modelos de aprendizagem com as experiências que temos vivenciado e cuja existência só é possível com o suporte das tecnologias inteligentes.

O trabalho está organizado da seguinte forma: a Seção 2 contém a fundamentação teórica, na Seção 3 discorremos sobre um modelo de aprendizagem sem fronteiras e na sequência, a Seção 4 traz a proposta de um framework conceitual com a discussão de um sistema de aprendizagem ubíquo. Finalmente, na Seção 5 apresentamos as considerações finais e trabalhos futuros.

\section{Fundamentação teórica}

Nesta seção serão abordados conceitos essenciais ao entendimento deste artigo, destacando-se as ecologias cognitivas, aprendizagem por interações, teoria epistemológica de Piaget e os modelos de aprendizagem correlatos.

Nós concordamos com a visão de [Smart et al. 2017] que a Internet pode ser vista como parte do ecossistema para a cognição humana. Ela serve como uma parte cada vez mais importante do ambiente material no qual um conjunto cada vez maior de atividades humanas cognitivas e epistêmicas se desdobra. Toda vez que fazemos upload, anotamos, editamos, twitamos ou postamos, estamos todos engajados na construção e configuração de um ambiente que (em retorno) desempenha um papel cada vez mais importante na formação do perfil de nossos esforços cognitivos e intelectuais.

A internet, bem como a inteligência artificial e as tecnologias digitais, estão assumindo um papel determinante no suporte às tarefas dos aprendizes, bem como no registro e na busca de informações e também no auxílio do percebimento ao que de fato importa (no que diz respeito a um determinado conteúdo) em um contexto onde muitas coisas acontecem ao mesmo tempo, tendo em vista que os indivíduos estão inseridos em diversos ecossistemas cognitivas simultaneamente. E são nesses pontos que a ubiquidade pode ajudar. 
VII Congresso Brasileiro de Informática na Educação (CBIE 2018)

Anais do XXIX Simpósio Brasileiro de Informática na Educação (SBIE 2018)

[Smart et al. 2017] acreditam que os ambientes cooperativos online, seja para ensino-aprendizagem ou sociais, estão imersos em um contexto onde as diversas formas de cognição podem ser exploradas ou potencializadas. O conjunto dessa manifestação de cognição são elementos constituintes das ecologias cognitivas, que podem ser definidas como "os contextos multidimensionais em que nos lembramos, sentimos, pensamos, comunicamos, imaginamos e agimos, com frequência em colaboração, em rica e contínua interação com nossos ambientes"[Tribble and Sutton 2011].

Esses conceitos evidenciam a importância que as interações têm no processo de construção do conhecimento. As formas de interação entre indivíduos possibilita trocar pontos de vista, conhecer e refletir sobre diferentes questionamentos e seu próprio pensar, e então ampliar com autonomia uma tomada de consciência para buscar novos rumos.

O desenvolvimento do pensamento requer um elemento social de cooperação. Partindo desse pressuposto, [Piaget 1973] define a cooperação como "operar em conjunto". Ela é caracterizada pela coordenação de diferentes pontos de vista, pela reciprocidade e pela existência de regras de condutas que têm por princípio o respeito bilateral.

[Perret-Clermont et al. 1995], baseado no trabalho de Piaget concentrouse na influência das interações sociais no desenvolvimento cognitivo, com a suposição que a aprendizagem acontece no interior de cada indivíduo, mas que é potencializada pelas trocas sociais, e atribui às interações um papel preponderante no desenvolvimento cognitivo do sujeito.

[Echeita and Martin 1995] destacam a importância da interação, dentro do contexto da aprendizagem cooperativa, no sentido que "o conhecimento é gerado, construído ou melhor dito, construído conjuntamente, porque se produz interatividade entre duas ou mais pessoas que participam dela". Esta interação social ou interpessoal é identificada como aspecto fundamental tanto pela epistemologia genética quanto pela escola sócio-histórica.

Nesse contexto é desejável a existência de recursos da tecnologia digital, inclusive a inteligência artificial, para apoiar o trabalho de estudantes e professores sob a perspectiva de ambientes que potencializem o processo de aprender independente do contexto específico de uma sala de aula, como no trabalho, na formação continuada, ou até mesmo no lazer e nas atividades do dia a dia, de forma que seja valorizado o fato de que independente do que esteja sendo feito, estamos sempre aprendendo.

A inteligência artificial aplicada à educação tem oferecido a possibilidade de aprender mais personalizado, visando fornecer aos professores e aprendizes as ferramentas que os permitam responder ao que está sendo aprendido e a como está sendo aprendido e como o aluno se sente no processo. Isso pode ajudar os aprendizes a construir o conhecimento que buscam [Luckin et al. 2016].

Identificamos na literatura alguns modelos de aprendizagem correlatos ao da aprendizagem sem fronteiras, no sentido de não ser limitado aos espaços, pelas disciplinas ou pelo contexto de atuação dos aprendentes (como está descrito 
VII Congresso Brasileiro de Informática na Educação (CBIE 2018)

Anais do XXIX Simpósio Brasileiro de Informática na Educação (SBIE 2018)

na seção 3.2). Dentre eles, citamos principalmente seamless learning (aprendizagem contínua), emergent learning (aprendizagem emergente) e ubiquitous learning (aprendizagem ubíqua).

Seamless Learning se apoia no entendimento que um aluno pode aprender sempre que estiver curioso em uma variedade de cenários e que pode alternar de um cenário para outro com facilidade e rapidez usando o dispositivo móvel pessoal como mediador. Esses cenários incluem a aprendizagem individual, com outro aluno, um pequeno grupo ou uma grande comunidade online, com possível envolvimento de professores, mentores, pais etc., frente a frente ou a distância, dentro e fora das salas de aula. A exploração e a investigação no espaço de Seamless Learning proporcionam um potencial para estender o tempo de aprendizagem formal, geralmente limitado à sala de aula, ao tempo de aprendizado informal, para abraçar oportunidades de aprendizagem fora da escola, impulsionadas pelos interesses pessoais dos alunos, o que pode envolver interação com uma comunidade geral, visitando museus, participando de projetos comunitários ou outros locais [Wong et al. 2015].

[Williams et al. 2011] definem Emergent Learning como uma aprendizagem que surge da interação entre um número de pessoas e recursos, na qual os aprendizes organizam e determinam tanto o processo quanto, em certa medida, os destinos de aprendizagem, ambos imprevisíveis. A interação é, em muitos sentidos, auto-organizada, mas requer, no entanto, alguma restrição e estrutura. Pode incluir redes de aprendizagem, virtuais ou físicas, ou ambas.

Ubiquitous learning é um paradigma educacional tornado possível em parte pelas possibilidades da mídia digital. [Cope and Kalantzis 2009] se propõem a explorar as dimensões dessa proposição e consequentemente um novo paradigma educacional. Esse paradigma é composto por algumas características, dentre as quais a primeira é relacionada a quebra das tradicionais fronteiras institucionais, espaciais e temporais da educação, de forma que a aprendizagem seja onipresente. O surgimento da computação ubíqua cria as condições para apoiar esse acontecimento.

\section{Em busca de uma aprendizagem sem fronteiras}

Nesta seção apresentaremos um breve panorama do atual estado de ensinoaprendizagem com os quais estamos acostumados na escola tradicional de acordo com alguns autores da área de educação e, em seguida, apresentaremos um contraponto com nossa proposta de potencialização da aprendizagem.

\subsection{A aprendizagem com fronteiras}

Fronteiras nada mais são que uma marcação imposta por alguém para determinar limites. Identificamos que nosso sistema de ensino tradicional estabeleceu cinco fronteiras principais: (i) Física: delimitada por um espaço e tempo específicos; (ii) Mesmo os ambientes digitais que ainda imitam a fronteira física, inclusive com utilização de senhas de acesso; (iii) Grade curricular formada por disciplinas; (iv) Tipo de atividades que estamos desempenhando: só se aprende quando estamos estudando tradicionalmente, entretanto podemos aprender durante atividades do dia a dia; (v) Determinação preemptória de quem ensina e quem aprende. 
VII Congresso Brasileiro de Informática na Educação (CBIE 2018)

Anais do XXIX Simpósio Brasileiro de Informática na Educação (SBIE 2018)

A prática de educação ainda é substancialmente moldada por modos de ensino tradicionais, resultado de aprendizagem transmissiva, expectativas normativas e relação hierárquica ao invés de relação heterárquica. A menos que as instituições, tanto na educação como no trabalho, ampliem seus espaços de aprendizagem para permitir maior flexibilidade e mais auto-organização, elas poderiam falhar em lidar com o dilema crescente que "mesmo quando os alunos estão na escola, grande parte de sua aprendizagem acontece fora dela" [Williams et al. 2011].

Há algum tempo, muitos pesquisadores buscam demonstrar as falhas do sistema educacional. Na década de noventa [Papert 1993] falava em repensar o modelo de educação vigente tendo em vista como a tecnologia se desenvolvia e o interesse dos estudantes se voltava cada vez mais para ela, partindo do pressuposto que a aprendizagem é atribuída a motivação e interesse dos indivíduos.

Mais recentemente, [Moraes 2008] mencionava em sua pesquisa que compreende que o grande problema da educação está na aplicação no modelo da ciência, que prevaleceu em um certo momento histórico, nas teorias de aprendizagem que o fundamentam e que influenciam a prática pedagógica. [Moraes 2008] ainda contesta que uma ciência do passado produz uma escola morta, dissociada da realidade, do mundo e da vida, e que uma educação sem vida produz seres incompetentes, incapazes de pensar, construir e reconstruir conhecimento.

A escola como conhecemos, ainda não está preparada para a revolução educacional que já começou há algum tempo. [Collins and Halverson 2018] descrevem que as pessoas ao redor do mundo estão tirando sua educação da escola e colocando em suas casas, bibliotecas, cafés e locais de trabalho, onde podem decidir o que querem aprender, quando querem aprender e como querem aprender. Isso desafia nosso modelo tradicional de educação com a aprendizagem à sala de aula. Esses novos nichos de aprendizagem usam tecnologias para permitir que pessoas de todas as idades busquem aprender em seus próprios termos, levando as pessoas a uma aprendizagem sem fronteiras.

\subsection{A aprendizagem sem fronteiras}

Não estamos propondo aqui um novo paradigma educacional. A aprendizagem sem fronteiras já existe, mas precisa ter sua existência evidenciada para ela possa ser potencializada. E é isso que estamos buscando. Uma forma de fazer isso é utilizar os meios computacionais que temos em mãos, principalmente a inteligência artificial e a ubiquidade oferecida pela tecnologia.

Aprender sem fronteiras advém do entendimento de que qualquer indivíduo é um aprendiz, tendo em vista, que estamos sempre aprendendo, em tudo o que fazemos e em qualquer lugar, contanto que exista interação com outros indivíduos ou com o ambiente.

Para superar as fronteiras, nos baseamos nos estudos realizados por [Chan et al. 2006] que se referem à aprendizagem contínua (seamless learning) com uma integração sinérgica das experiências de aprendizagem em várias dimensões, tais como contextos formais e informais de aprendizagem, individual e social, no mundo físico e no ciberespaço. A premissa básica da aprendizagem 
VII Congresso Brasileiro de Informática na Educação (CBIE 2018)

Anais do XXIX Simpósio Brasileiro de Informática na Educação (SBIE 2018)

contínuo é que não é factível nem produtivo aparamentar os aprendizes com todo o conhecimento e habilidades com base no período, local ou cenário de uma aprendizagem episódica, tal qual ocorre na educação formal [Chen et al. 2010].

A tecnologia móvel, que em nosso contexto é o principal componente da ubiquidade tecnológica que nos referimos, tem o potencial de mediar a aprendizagem contínua [Looi et al. 2010]. Embora a aprendizagem formal seja baseado em currículos fixos promulgados em ambientes de sala de aula, a informal acontece quando os alunos participam de experiências intencionais ou não intencionais fora do ambiente escolar, de forma que as duas formas de aprendizagem não devem ser vistas como situações dicotômicas e conflitantes [Sharples 2006].

De acordo com [Banks 1994], quando se fala de desenvolvimento do conhecimento em termos genéticos, [Piaget and Del Val 1970] consideram que próprio indivíduo tenha a responsabilidade na construção de novos conhecimentos. Ao mencionar o papel do sujeito em sua aprendizagem há de se levar em consideração uma outra conjectura de base no construtivismo, que trata o conhecimento não como uma cópia ou uma absorção daquilo que existe, mas como uma reestruturação, na qual intervêm o objeto e o sujeito com os seus esquemas de assimilação e acomodação. Em outras palavras, o conhecimento se constrói por meio das interações sujeito-objeto.

É importante levar em conta que interações humanas geram cognição e cognição gera conhecimento. No entanto, é imprescindível ter em conta que as interações compõem a base da existência das ecossistemas cognitivos. Nesta direção, faz sentido pensar que qualquer ser vivo que pode interagir, pode aprender, independente de escola, de professor ou de tecnologias. De fato as ecologias cognitivas não dependem da escola tradicional e nem mesmo das tecnologias para existirem, mas podem ser potencializadas por ambas.

Outra base de nossa proposta, para superar as fronteiras destacadas na seção anterior, tem raízes no paradigma ubiquitous learning (aprendizagem onipresente), também conhecido como u-learning, que promove em sua estrutura a utilização de tecnologia ubíqua. A ubiquidade é a característica mais significativa das tecnologias sem fio e móvel, e possibilita que os estudantes aprendam a coisa certa, no momento certo, no lugar certo [Peng et al. 2009].

No fim das contas, a aprendizagem sem fronteiras, se refere a um modo de aprender no qual o estudante não está limitado às paredes da escola tradicional e não necessariamente sobre um curriculo específico. Ele aprende na escola, em casa, no museu, no zoológico ou onde quer que esteja e de forma contínua com o apoio, principalmente, da tecnologia móvel que nos acompanha praticamente em todos os lugares e da inteligência artificial à serviço do estudante, atuando na verificação dos caminhos percorridos e indicando alternativas, e se for o caso, o ajudando na transição e compartilhamento de informações nos diversos ecossistemas cognitivos em que ele estiver presente.

\section{Uma proposta conceitual para fomentar ecologias cognitivas}

Aqui apresentaremos um framework conceitual para potencializar a aprendizagem sem fronteiras na fomentação de um ecossistema cognitivo em conjunto com 
VII Congresso Brasileiro de Informática na Educação (CBIE 2018)

Anais do XXIX Simpósio Brasileiro de Informática na Educação (SBIE 2018)

uma prova de conceito, em forma de instância desse framework. Trata-se do Laboratório de Informática na Educação Ubíquo ou ainda LIEd Ubíquo.

Os indivíduos desse laboratório estão interagindo em eventos em seu cotidiano a todo tempo. Essas pessoas participam de aulas e fazem trabalhos escolares, individuais e coletivos de forma que mesmo quando o trabalho é individual há uma rede de interações. A Figura 1 mostra esse panorama.

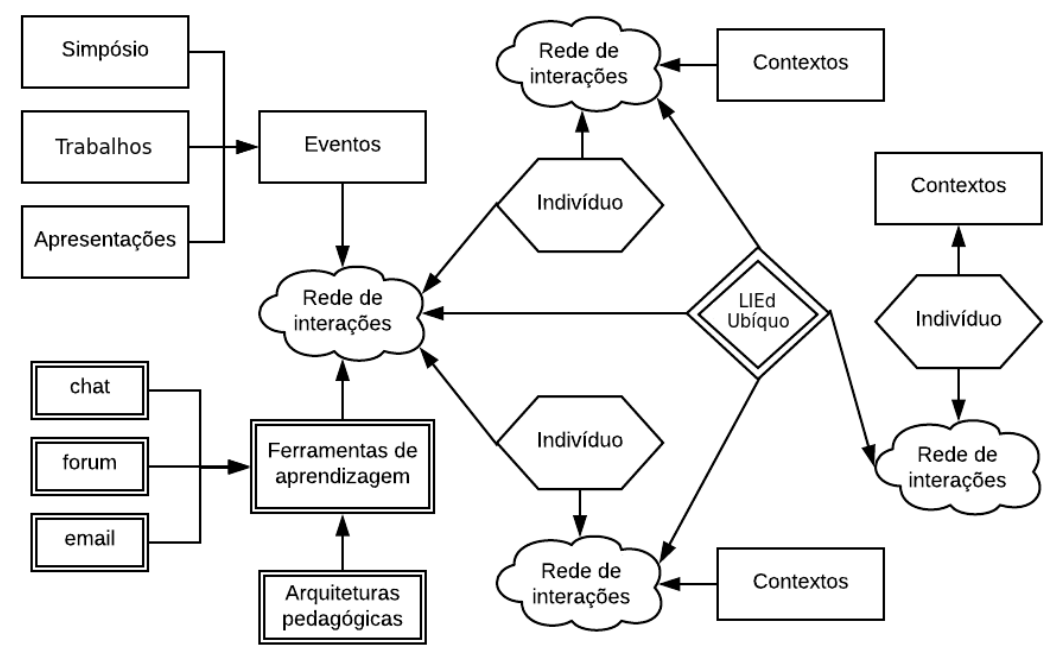

Figura 1. Framework conceitual sob o ponto de vista das interações

No âmbito do laboratório são criadas teses, dissertações, tcc, experimentos, artigos e diferentes projetos. Essas produções são apresentadas em eventos e em torno deles são construídas várias interações que favorecem a construção de conhecimento. Assistir a uma apresentação, desperta dúvidas e gera interações entre os "ouvintes"e destes com os apresentadores. E ainda leva a discussões com outros pares mesmo que não sejam estudantes do laboratório.

Em torno de um membro do laboratório existem outras redes das quais ele participa e para onde essas conversas podem fluir e quando um participante fala com um amigo sobre o que está fazendo, ele também traz algo similar de um outro contexto, que pode ser familiar, círculo de amizades, trabalho etc. Esse cruzamento pode facilmente se perder e as instâncias do framework que propomos são recursos que podem ajudar a evitar que isso aconteça. Dessa forma, a preocupação sobre a construção do conhecimento não deve ser sobre a escola mas sobre as comunidades de aprendizagem.

O LIEd Ubíquo é uma primeira instância desse framework. Se trata de uma ferramenta de apoio à construção do conhecimento no contexto de um ecossistema cognitivo cujos participantes são agentes artificiais e humanos de um mesmo laboratório de pesquisa. Grande parte das produções, interações e discussões deverão ser contempladas nesta solução que deve ser acessível de qualquer lugar e de qualquer dispositivo.

A Figura 2 mostra um exemplo de alguns indivíduos e os ecossistemas cognitivos nos quais estão inseridos, com a intenção de detalhar os componentes do LIEd Ubíquo e como acontece a ligação dele com um ecossistema cognitivo 
VII Congresso Brasileiro de Informática na Educação (CBIE 2018)

Anais do XXIX Simpósio Brasileiro de Informática na Educação (SBIE 2018)

coletivo construído a partir de interesses comuns.

Este exemplo demonstra um ecossistema composto por ecossistemas de dois tipos: individuais, com interações "indivíduo x ambiente", de forma que cada individuo tem uma ecologia particular, construída pela seleção dos vários ecossistemas dos quais participa. E ainda um ecossistema coletivo (que engloba um ambiente de trabalho e um escolar) com interações "indivíduo $x$ ambiente $x$ indivíduo".

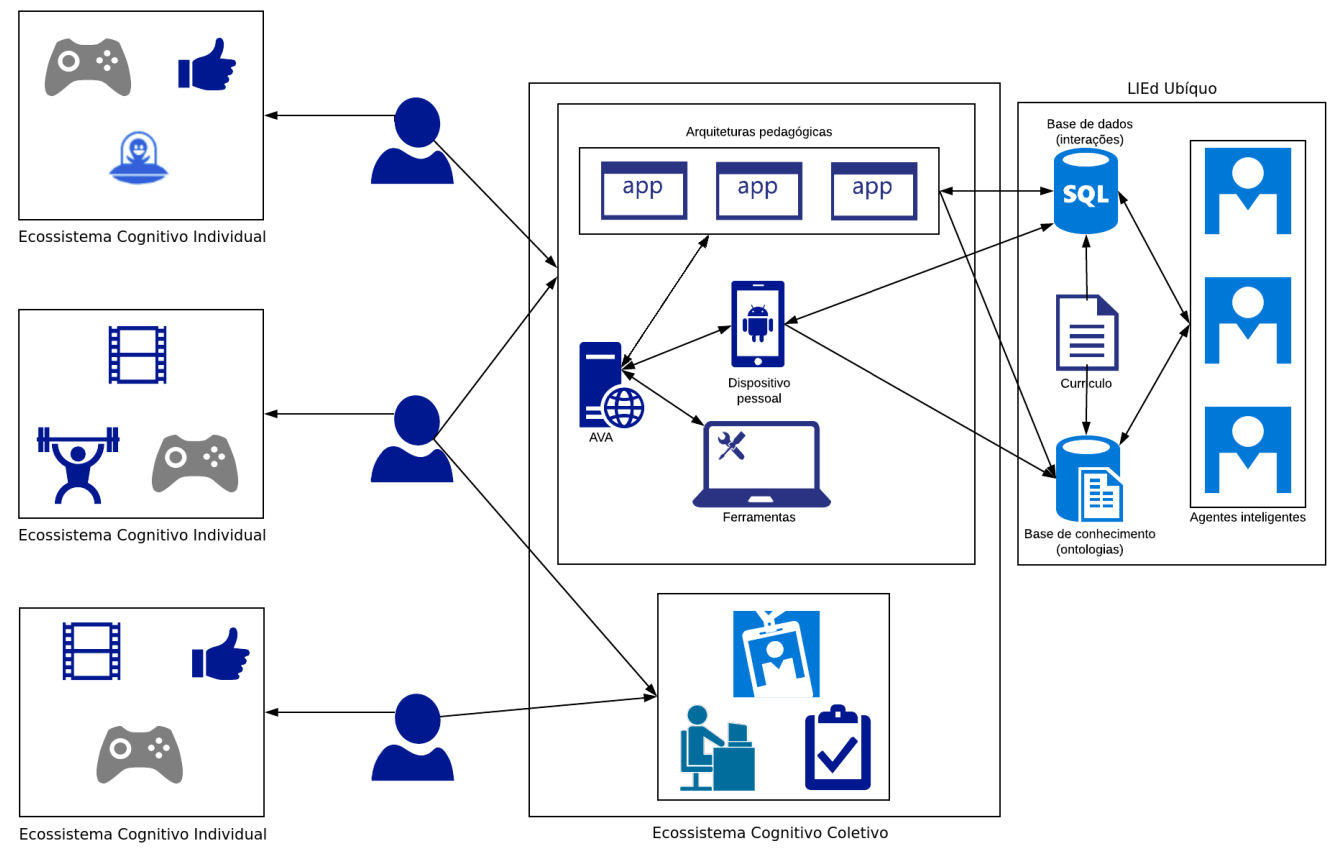

Figura 2. Ecossistema de aprendizagem com detalhe do LIEd Ubíquo

Como suporte para construção do conhecimento, o aprendiz tem as ferramentas (editores de texto, planilhas, construtores de site etc) e as arquiteturas pedagógicas [Carvalho et al. 2005] no curso de disciplinas ou no dia-a-dia que geram, a partir das interações, a principal fonte de dados para análises dos agentes, bem como informações de currículo, institucionais, de utilização de ambientes virtuais de aprendizagem, dentre outras.

O LIEd Ubíquo, em termos tecnológicos, depende de alguns agentes inteligentes e de alguns depósitos de dados: um banco de dados padrão e uma ontologia de domínio. Essa proposta atua diretamente no ecossistema formado no laboratório, mas os agentes também capturam dados das outros ecossistemas onde o estudante esteja inserido e pode interferir com serviço de suporte à aprendizagem (a partir dos dispositivos móveis) caso detectem que o indivíduo esteja envolvido com alguma atividade relacionada a conteúdo prioritário. Alguns exemplos disso são: sugestão de leituras, sugestão de aproximação com outros indivíduos, alertas sobre tempo de atividades dentre outros.

A ferramenta tem o intuito de ser ubíqua não apenas no sentido do "sensível ao contexto" mas buscando de fato a onipresença, como um assistente (individual ou coletivo) daqueles que estejam envolvidos no processo de apren- 
VII Congresso Brasileiro de Informática na Educação (CBIE 2018)

Anais do XXIX Simpósio Brasileiro de Informática na Educação (SBIE 2018)

dizagem cooperativa, permitindo registros, fazendo análises e principalmente favorecendo interações.

Ainda podemos citar, como finalidades do sistema: (i) manter uma base de conhecimento com informações obtidas do portfólio, interações, redes sociais, e-mail e currículo lattes, (ii) promover a cooperação entre os integrantes do laboratório segundo suas áreas de interesse e (iii) apresentar novidades registradas no portfólio de aprendizagem dos integrantes do laboratório, propondo aproximação de indivíduos e grupos.

Por fim, acreditamos que o LIEd Ubíquo possa funcionar como um assistente pessoal e/ou de grupo, sempre ao lado dos aprendizes, ajudando-os na recomendação de conteúdos, respondendo questionamentos e armazenando suas ideias, características e dados educacionais gerados por eles mesmos e por sistemas que estejam utilizando.

\section{Considerações finais}

Neste artigo destacamos um modelo de aprendizagem nomeado como "sem fronteiras" que possui fortes relações com as ecologias cognitivas e apresentamos um framework conceitual para fomentá-lo. Em nossa proposta, os estudantes aprendem de forma contínua, dentro e fora da escola tradicional e com o apoio da inteligência artificial, da tecnologia ubíqua e da internet.

Nossa primeira contribuição está na apresentação e estruturação desse paradigma de aprendizagem o qual vem sendo discutido por diversos estudiosos há algum tempo, com outras nomenclaturas. A segunda contribuição contempla a disponibilização de um modelo para potencializar a aprendizagem sem fronteiras, considerando aspectos teóricos e tecnológicos.

Como principal trabalho futuro, é considerada a programação da ferramenta LIEd Ubíquo e a obtenção de evidências de aprendizagem com a execução prática e empírica do paradigma que já está consolidado no campo teórico.

\section{Referências}

Banks, L. (1994). As interações sociais na perspectiva piagetiana. Série Idéias, São Paulo, 20:41-47.

Carvalho, M. J. S., de Nevado, R. A., and de Menezes, C. S. (2005). Arquiteturas pedagógicas para educação à distância: concepções e suporte telemático. In Brazilian Symposium on Computers in Education (Simpósio Brasileiro de Informática na Educação-SBIE), volume 1, pages 351-360.

Chan, T.-W., Roschelle, J., Hsi, S., Kinshuk, Sharples, M., Brown, T., Patton, C., Cherniavsky, J., PEA, R., Norris, C., et al. (2006). One-to-one technologyenhanced learning: An opportunity for global research collaboration. Research and Practice in Technology Enhanced Learning, 1(01):3-29.

Chen, W., Seow, P., So, H.-J., Toh, Y., and Looi, C.-K. (2010). Extending students' learning spaces: technology-supported seamless learning. In Proceedings of the 9th International Conference of the Learning Sciences-Volume 1, pages 484-491. International Society of the Learning Sciences. 
VII Congresso Brasileiro de Informática na Educação (CBIE 2018)

Anais do XXIX Simpósio Brasileiro de Informática na Educação (SBIE 2018)

Collins, A. and Halverson, R. (2018). Rethinking education in the age of technology: The digital revolution and schooling in America. Teachers College Press.

Cope, B. and Kalantzis, M. (2009). Ubiquitous learning: An agenda for educational transformation. Ubiquitous learning, pages 3-14.

Echeita, G. and Martin, E. (1995). Interação social e aprendizagem. Desenvolvimento Psicológico e educação: necessidades educativas especiais e aprendizagem escolar. Porto Alegre: Artes Médicas, 3:36-53.

Lévy, P. (1999). As tecnologias da inteligência: o futuro do pensamento na era da informática. rio de janeiro: Editora 34, 1993. Coleção Trans.

Looi, C.-K., Seow, P., Zhang, B., So, H.-J., Chen, W., and Wong, L.-H. (2010). Leveraging mobile technology for sustainable seamless learning: a research agenda. British journal of educational technology, 41(2):154-169.

Luckin, R., Holmes, W., Griffiths, M., and Forcier, L. B. (2016). Intelligence unleashed: An argument for ai in education.

Moraes, M. C. (2008). O paradigma educacional emergente: implicações na formação do professor e nas práticas pedagógicas. Em aberto, 16(70).

Papert, S. (1993). The children's machine: Rethinking school in the age of the computer. ERIC.

Peng, H., Su, Y.-J., Chou, C., and Tsai, C.-C. (2009). Ubiquitous knowledge construction: Mobile learning re-defined and a conceptual framework. Innovations in Education and Teaching international, 46(2):171-183.

Perret-Clermont, A.-N., Godinho, E., Gaspar, F. L., and Gaspar, C. (1995). Desenvolvimento da inteligência e interaç̧ão social.

Piaget, J. (1973). As operações lógicas e a vida social. Estudos sociológicos, pages 164-193.

Piaget, J. and Del Val, J. A. (1970). La epistemología genética. A. Redondo.

Sharples, M. (2006). How can we address the conflicts between personal informal learning and traditional classroom education. Big issues in mobile learning, pages 21-24.

Smart, P., Heersmink, R., and Clowes, R. W. (2017). The cognitive ecology of the internet. In Cognition beyond the brain, pages 251-282. Springer.

Tribble, E. and Sutton, J. (2011). Cognitive ecology as a framework for shakespearean studies. Shakespeare Studies, 39:94.

Williams, R., Karousou, R., and Mackness, J. (2011). Emergent learning and learning ecologies in web 2.0. The International Review of Research in Open and Distributed Learning, 12(3):39-59.

Wong, L.-H., Milrad, M., and Specht, M. (2015). Seamless learning in the age of mobile connectivity. Springer. 